\title{
BECKER-GOTTLIEB TRANSFER FOR HOCHSCHILD COHOMOLOGY
}

\author{
FEI XU
}

(Communicated by Pham Huu Tiep)

\begin{abstract}
Let $G$ be a finite group. Over any finite $G$-poset $\mathcal{P}$ we may define a transporter category $G \propto \mathcal{P}$ as the corresponding Grothendieck construction. There exists a Becker-Gottlieb transfer from the ordinary cohomology of $G \propto \mathcal{P}$ to that of $G$. We shall construct it using module-theoretic methods and then extend it to a transfer from the Hochschild cohomology of $k(G \propto \mathcal{P})$ to that of $k G$, where $k$ is a base field.
\end{abstract}

\section{INTRODUCTION}

Let $G$ be a finite group, $\mathcal{P}$ a finite $G$-poset and $k$ a field. Here we always consider $G$ as a category with one object $\bullet$. Then having a $G$-poset $\mathcal{P}$ means there exists a functor $F$ from $G$ to the category of small categories such that $F(\bullet)=\mathcal{P}$. The Grothendieck construction on $F$ is a finite category and is written as $G \propto \mathcal{P}$, equipped with a canonical functor $\pi: G \propto \mathcal{P} \rightarrow G$. We may call such a category a transporter category, as the usual transporter categories can be introduced in this way. Our concept is more general because moreover each subgroup $H$ of $G$ can be recovered as a transporter category $G \propto(G / H)$, for the $G$-set $G / H$, up to a category equivalence. It makes sense if we deem transporter categories as generalized subgroups for a fixed finite group. Topologically it is well known that $B(G \propto \mathcal{P}) \simeq E G \times_{G} B \mathcal{P}$, and in particular $B(G \propto(G / H)) \simeq B H$. Forming the transporter category over a $G$-poset eliminates the $G$-action and thus is the algebraic analogy of introducing a Borel construction over a $G$-space. In this article we shall study the connections between $G$ and $G \propto \mathcal{P}$ through their category algebras $k G$ and $k(G \propto \mathcal{P})$; see Section 2.3.

For any subgroup $H$ and two $k G$-modules $M$ and $N$, there is a transfer map

$$
\operatorname{tr}_{H}^{G}: \operatorname{Ext}_{k H}^{*}\left(M \downarrow_{H}^{G}, N \downarrow_{H}^{G}\right) \rightarrow \operatorname{Ext}_{k G}^{*}(M, N) .
$$

It can be generalized to a Becker-Gottlieb transfer map

$$
\operatorname{tr}_{\mathcal{P}}: \operatorname{Ext}_{k(G \propto \mathcal{P})}^{*}\left(\kappa_{M}, \kappa_{N}\right) \rightarrow \operatorname{Ext}_{k G}^{*}(M, N),
$$

where $\kappa_{M}$ and $\kappa_{N}$ are the restrictions of $M$ and $N$, respectively. We shall construct the Becker-Gottlieb transfer in a way parallel to the previous one using moduletheoretic methods, borrowing ideas from Dwyer-Wilkerson [6]. In this greater generality, $\operatorname{tr}_{G / H}=\operatorname{tr}_{H}^{G}$.

Received by the editors April 3, 2012 and, in revised form, August 11, 2012.

2010 Mathematics Subject Classification. Primary 20C05; Secondary 20J99.

The author (徐斐) was supported in part by a Beatriu de Pinós research fellowship from the government of Catalonia of Spain. 
Using similar methods we will construct another transfer map between Hochschild cohomology.

Theorem 1.1. Let $\pi: G \propto \mathcal{P} \rightarrow G$ be the canonical functor. Suppose $\operatorname{Res}_{\pi}: k G$ $\bmod \rightarrow k(G \propto \mathcal{P})$-mod is the restriction along $\pi$ and write $\kappa_{M}=\operatorname{Res}_{\pi} M$ for any $M \in k G$-mod. Then:

(1) we have the following two maps, restriction and transfer,

$$
\operatorname{Ext}_{k G}^{*}(M, N) \stackrel{\text { resp }}{\rightarrow} \operatorname{Ext}_{k(G \propto \mathcal{P})}^{*}\left(\kappa_{M}, \kappa_{N}\right) \stackrel{\mathrm{tr}_{\mathcal{P}}}{\rightarrow} \operatorname{Ext}_{k G}^{*}(M, N),
$$

which compose to $\chi(\mathcal{P} ; k) \cdot 1$, multiplication by the Euler characteristic of (the order complex of) $\mathcal{P}$, and

(2) there exists a map

$$
\operatorname{htr}_{\mathcal{P}}: \operatorname{Ext}_{k(G \propto \mathcal{P})^{e}}^{*}(k(G \propto \mathcal{P}), k(G \propto \mathcal{P})) \rightarrow \operatorname{Ext}_{k G^{e}}^{*}(k G, k G)
$$

which induces $\operatorname{tr}_{\mathcal{P}}: \operatorname{Ext}_{k(G \propto \mathcal{P})}^{*}(\underline{k}, \underline{k}) \rightarrow \operatorname{Ext}_{k G}^{*}(k, k)$.

Here $\underline{k}$ is $\kappa_{k}$, the trivial $k(G \propto \mathcal{P})$-module, and is of great importance. We comment that (2) makes sense because $\operatorname{Ext}_{k \mathcal{C}}^{*}(\underline{k}, \underline{k})$ is a direct summand of $\operatorname{Ext}_{k \mathcal{C}^{e}}^{*}(k \mathcal{C}, k \mathcal{C})$ [14]. In particular, it generalizes a transfer map of Linckelmann for $\mathcal{P}=G / H$ [ 8 .

For other information about $\operatorname{Ext}_{k(G \propto \mathcal{P})}^{*}(\mathfrak{M}, \mathfrak{N})$, where $\mathfrak{M}, \mathfrak{N} \in k(G \propto \mathcal{P})$-mod are not necessarily restrictions of $k G$-modules, the reader is referred to [16].

\section{Preliminaries}

In this section, we recall the definition of a transporter category and some background in category algebras. Throughout this article we will only consider finite categories, in the sense that they have finitely many morphisms. Thus a group $G$, or a $G$-poset $\mathcal{P}$, is always finite.

Although many constructions can be made over a commutative ring with identity, we work throughout this article over a field $k$ for convenience.

2.1. Transporter categories as Grothendieck constructions. We usually deem a group as a category with one object, usually denoted by $\bullet$. The identity of a group is named $e$. We say a poset $\mathcal{P}$ is a $G$-poset if there exists a functor $F$ from $G$ to $s C a t s$, the category of small categories, such that $F(\bullet)=\mathcal{P}$. The Grothendieck construction on $F$ is called a transporter category. In the following explicit definition, the morphisms in a poset are customarily denoted by $\leq$.

Definition 2.1. Let $G$ be a group and $\mathcal{P}$ a $G$-poset. The transporter category $G \propto \mathcal{P}$ has the same objects as $\mathcal{P}$, that is, $\mathrm{Ob}(G \propto \mathcal{P})=\mathrm{Ob} \mathcal{P}$. For $x, y \in \operatorname{Ob}(G \propto$ $\mathcal{P})$, a morphism from $x$ to $y$ is a pair $(g, g x \leq y)$ for some $g \in G$.

The symbol $G \propto \mathcal{P}$ is used because this particular Grothendieck construction resembles a semidirect product, yet is different. From the definition one can easily see that there is a natural embedding $\iota_{\mathcal{P}}: \mathcal{P} \hookrightarrow G \propto \mathcal{P}$ via $(x \leq y) \mapsto(e, x \leq y)$. On the other hand, the transporter category admits a natural functor $\pi_{\mathcal{P}}: G \propto \mathcal{P} \rightarrow G$, given by $x \mapsto \bullet$ and $(g, g x \leq y) \mapsto g$. The functor $\pi_{\mathcal{P}}$ is obtained by functoriality of 
the Grothendieck construction via the unique functor $\mathcal{P} \rightarrow \bullet$ and the isomorphism $G \propto \bullet \cong G$. We always have a sequence of functors

$$
\mathcal{P} \stackrel{\iota_{\mathcal{P}}}{\rightarrow} G \propto \mathcal{P} \stackrel{\pi_{\mathcal{P}}}{\rightarrow} G
$$

such that $\pi_{\mathcal{P}} \circ \iota_{\mathcal{P}}(\mathcal{P})$ is the trivial subgroup or subcategory of $G$. For convenience, in the rest of this article we often neglect the subscript $\mathcal{P}$ and write $\iota=\iota_{\mathcal{P}}, \pi=\pi_{\mathcal{P}}$. The classifying spaces of the above sequence form a fibration

$$
B \mathcal{P} \rightarrow B(G \propto \mathcal{P}) \cong E G \times{ }_{G} \mathcal{P} \rightarrow B G .
$$

Example 2.2. If $G$ acts trivially on $\mathcal{P}$, then $G \propto \mathcal{P}=G \times \mathcal{P}$.

Example 2.3. Let $G$ be a finite group and $H$ a subgroup. We consider the set of left cosets $G / H$ which can be regarded as a $G$-poset: $G$ acts via left multiplication. The transporter category $G \propto(G / H)$ is a connected groupoid whose skeleton is isomorphic to $H$. In this way one can recover all subgroups of $G$, up to category equivalences.

A category equivalence $\mathcal{D} \rightarrow \mathcal{C}$ induces a Morita equivalence between the category algebras (to be recalled in Section 2.3) $k \mathcal{D} \simeq k \mathcal{C}$ (see [13), as well as a homotopy equivalence $B \mathcal{D} \simeq B \mathcal{C}$. It means there is no essential difference between $k H$ and $k(G \propto(G / H))$ (and their module categories) or between $B H$ and $B(G \propto(G / H))$.

2.2. Nerves and homology representations of $k G$. Suppose $\mathcal{C}$ is a small category. Its nerve $N \mathcal{C}$ is a simplicial set, from which one can construct a (normalized) chain complex $\mathbb{C}_{*}(\mathcal{C}, k)$ such that, for each $i>0, \mathbb{C}_{i}(\mathcal{C}, k)$ is a $k$-vector space with a basis the set of $i$-chains of (non-identity) morphisms in $\mathcal{C}$, while $\mathbb{C}_{0}(\mathcal{C}, k)=k \mathrm{Ob} \mathcal{C}$. The topological realization of $N \mathcal{C}$ is called the classifying space of $\mathcal{C}$ and is written as $B \mathcal{C}$. The homology of the complex $\mathbb{C}_{*}(\mathcal{C}, k)$ gives rise to the homology of $B \mathcal{C}$, i.e. $\mathrm{H}_{*}(B \mathcal{C}, k)$. The $k$-dual, $\mathbb{C}_{*}(\mathcal{C}, k)^{\wedge}$, calculates $\mathrm{H}^{*}(B \mathcal{C}, k)$, the singular cohomology of $B \mathcal{C}$. The Euler characteristic $\chi(\mathcal{C})=\chi(\mathcal{C}, k)$ is defined to be $\sum_{i>0}(-1)^{i} \operatorname{dim}_{k} \mathbb{C}_{i}(\mathcal{C}, k)$, assuming $\mathbb{C}_{*}(\mathcal{C}, k)$ is a finite complex.

When $\mathcal{C}$ admits a $G$-action, $\mathbb{C}_{*}(\mathcal{C}, k)$ becomes a complex of permutation $k G$ modules. The homology and cohomology groups of $B \mathcal{C}$ are also $k G$-modules. This way of constructing $k G$-modules has been studied intensively when the category is a $G$-poset. In the present article we shall mainly focus on constructing $k G$-modules from $G \propto \mathcal{P}$, which is closely related to certain constructions over $\mathbb{C}_{*}(\mathcal{P}, k)$.

Example 2.4. In Example 2.3, $\mathbb{C}_{*}(G / H)$ is a stalk complex $\mathbb{C}_{*}(G / H)=\mathbb{C}_{0}(G / H)$ $\cong k \uparrow_{H}^{G}$ with $\chi(G / H)=[G: H]$.

2.3. Category algebras, representations and cohomology. We recall some facts about category algebras. The reader is referred to [13, 15, for further details. Let $\mathcal{C}$ be a finite category and $k$ a field. One can define category algebra $k \mathcal{C}$, which has as a basis the set of all morphisms in $\mathcal{C}$. The multiplication is given by compositions of base elements. When $\mathcal{C}$ is a group, $k \mathcal{C}$ is exactly the group algebra. A $k$-representation of $\mathcal{C}$ is a covariant functor from $\mathcal{C}$ to $V e c t_{k}$, the category of finite dimensional $k$-vector spaces. All representations of $\mathcal{C}$ form the functor category $V e c t_{k}^{\mathcal{C}}$. By a theorem of B. Mitchell, the finitely generated left $k \mathcal{C}$-modules are the same as the $k$-representations of $\mathcal{C}$, in the sense that there exists a natural equivalence

$$
V e c t_{k}^{\mathcal{C}} \simeq k \mathcal{C}-\bmod
$$


In the module category, there is a distinguished module $\underline{k}$, sometimes called the trivial module, which can be defined as a constant functor taking $k$ as its value at every object of $\mathcal{C}$. Since $V e c t_{k}$ is a symmetric monoidal category, $V e c t_{k}^{\mathcal{C}}$ inherits this structure. There exists an (internal) tensor product, or the pointwise tensor product, written as $\hat{\otimes}$, such that for any two $k \mathcal{C}$-modules $\mathfrak{M}, \mathfrak{N},(\mathfrak{M} \hat{\otimes} \mathfrak{N})(x)=$ $\mathfrak{M}(x) \otimes_{k} \mathfrak{N}(x)$. If $\alpha \in \operatorname{Mor} \mathcal{C}$ is a base element in $k \mathcal{C}$, then $\alpha$ acts on $\mathfrak{M} \hat{\otimes} \mathfrak{N}$ via $\alpha \otimes \alpha$. Obviously $\underline{k}$ is the identity with respect to $\hat{\otimes}$ and $\mathfrak{M} \hat{\otimes} \mathfrak{N} \cong \mathfrak{N} \hat{\otimes} \mathfrak{M}$. We can also construct a function object, the internal hom, $\mathcal{H o m}(\mathfrak{M}, \mathfrak{N}) \in k \mathcal{C}$-mod such that

$$
\operatorname{Hom}_{k \mathcal{C}}(\mathfrak{L} \hat{\otimes} \mathfrak{M}, \mathfrak{N}) \cong \operatorname{Hom}_{k \mathcal{C}}(\mathfrak{L}, \mathcal{H o m}(\mathfrak{M}, \mathfrak{N}))
$$

for any $\mathfrak{L} \in k \mathcal{C}$-mod, and the isomorphism is natural in $\mathfrak{M}$ and $\mathfrak{N}$. The monoidal structure allows us to define a cup product on $\operatorname{Ext}_{k \mathcal{C}}^{*}(\underline{k}, \underline{k})$. The resulting ring is isomorphic to $\mathrm{H}^{*}(B \mathcal{C}, k)$ in a natural way.

The following functors for comparing category algebras and their modules play a key role in our constructions of transfer maps. When $\tau: \mathcal{D} \rightarrow \mathcal{C}$ is a functor between two finite categories. There are functors for comparing these representations. The functor $\tau$ induces a restriction

$$
\operatorname{Res}_{\tau}: k \mathcal{C} \text {-mod } \rightarrow k \mathcal{D} \text {-mod. }
$$

If we regard a $\mathrm{kC}$-module as a functor, then its restriction is the precomposition of it with $\tau$. The functor $\operatorname{Res}_{\tau}$ is equipped with two adjoints: the left and right Kan extensions

$$
L K_{\tau}, R K_{\tau}: k \mathcal{D} \text {-mod } \rightarrow k \mathcal{C} \text {-mod. }
$$

The definition of the left and right Kan extensions depend on the so-called overcategories and undercategories, respectively. For each $x \in \mathrm{Ob} \mathcal{C}$, one can define an overcategory $\tau / x$ whose objects are pairs $\left\{(d, \alpha) \mid \alpha \in \operatorname{Hom}_{\mathcal{C}}(\tau(d), x)\right\}$. A morphism from $(d, \alpha)$ to some $\left(d^{\prime}, \beta\right)$ is a morphism $f \in \operatorname{Hom}_{\mathcal{D}}\left(d, d^{\prime}\right)$ such that the following diagram commutes:

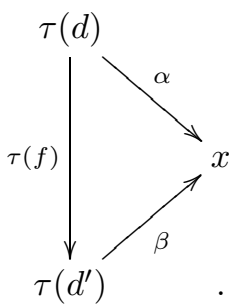

One can always build a canonical projective resolution $\mathcal{B}_{*}^{\mathcal{C}}$, the bar resolution, of $\underline{k} \in k \mathcal{C}$-mod. For each $n \geq 0, \mathcal{B}_{n}^{\mathcal{C}}:=\mathbb{C}_{n}\left(\operatorname{Id}_{\mathcal{C}} /-, k\right)$. It means, for each $x \in \mathrm{Ob} \mathcal{C}$, $\overline{\mathcal{B}}_{n}^{\mathcal{C}}(x)=\mathbb{C}_{n}\left(\operatorname{Id}_{\mathcal{C}} / x, k\right)$ is the $k$-vector space spanned over the $n$-chains of morphisms in $\operatorname{Id}_{\mathcal{C}} / x$. A well known fact is that, given a functor $\tau: \mathcal{D} \rightarrow \mathcal{C}$,

$$
L K_{\tau} \mathcal{B}_{*}^{\mathcal{D}} \cong \mathbb{C}_{*}(\tau /-, k)
$$

is a (usually not exact) complex of projective $k \mathcal{C}$-modules. To deal with category cohomology, one often has to understand the structure of such $L K_{\tau} \mathcal{B}_{*}^{\mathcal{D}}$ for various $\tau: \mathcal{D} \rightarrow \mathcal{C}$. The reader will see that calculating the structure of various chain complexes like $\mathbb{C}_{*}(\tau /-, k)$ is our major method in this article. In fact, we do so by analyzing the structure of those overcategories $\tau / x, x \in \mathrm{Ob} \mathcal{C}$, which determine 
the chain complexes. For convenience we often omit the base field in these chain complexes.

2.4. Factorization category and Hochschild cohomology. Let $\mathcal{C}$ be a finite category. We can consider its Hochschild cohomology $\operatorname{HH}^{*}(k \mathcal{C})=\operatorname{Ext}_{k \mathcal{C}}^{*}(k \mathcal{C}, k \mathcal{C})$, where $\mathcal{C}^{e}=\mathcal{C} \times \mathcal{C}^{o p}$ and $k \mathcal{C}^{e}$ is the enveloping algebra of $k \mathcal{C}$. The $k \mathcal{C}^{e}$-module $k \mathcal{C}$ as a functor $\mathcal{C}^{e} \rightarrow$ Vect $_{k}$ is given by

$$
k \mathcal{C}(x, y)=k \operatorname{Hom}_{\mathcal{C}}(y, x), \forall(x, y) \in \mathrm{ObC}^{e} .
$$

In [14] we proved that the Hochschild cohomology of $k \mathcal{C}$ is closely related to the ordinary cohomology. In order to state the result we need the category of factorizations in $\mathcal{C}$, written as $F(\mathcal{C})$. The objects are the morphisms in $\mathcal{C}$, and in order to avoid confusion, if a morphism $\alpha \in \operatorname{Mor} \mathcal{C}$ is considered as an object in $F(\mathcal{C})$, we shall denote it by $[\alpha] \in \operatorname{Ob} F(\mathcal{C})$. Then a morphism $[\alpha] \rightarrow[\beta]$ is a pair $(u, v)$ of morphisms in $\mathcal{C}$ such that $\beta=u \alpha v$. The category $F(\mathcal{C})$ plays the role of the diagonal subgroup $\Delta G \subset G \times G \cong G^{e}$ in group cohomology. There exists a commutative diagram of functors

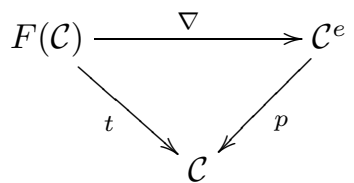

On objects the functor $t$ is given by $t([\alpha])=y$, if $\alpha \in \operatorname{Hom}_{\mathcal{C}}(x, y)$, the functor $\nabla$ is given by $\nabla([\alpha])=(y, x)$ and $p$ is the projection onto the first component. One can easily figure out their actions on morphisms. By using relevant restriction and Kan extensions along these functors we obtained the following isomorphisms [14]:

$$
\operatorname{Ext}_{k F(\mathcal{C})}^{*}(\underline{k}, \underline{k}) \cong \operatorname{Ext}_{k \mathcal{C}}^{*}(\underline{k}, \underline{k})
$$

(particularly, $t$ induces a homotopy equivalence $B F(\mathcal{C}) \simeq B \mathcal{C}$ ) and

$$
\operatorname{Ext}_{k \mathcal{C} e}^{*}(k \mathcal{C}, k \mathcal{C}) \cong \operatorname{Ext}_{k F(\mathcal{C})}^{*}\left(\underline{k}, \operatorname{Res}_{\nabla} k \mathcal{C}\right) .
$$

Moreover, Res $\nabla k \mathcal{C}$ has $\underline{k}$ as a direct summand and thus the ordinary cohomology is a direct summand of the Hochschild cohomology.

For future reference, we record the following result.

Lemma 2.5. If $\tau: \mathcal{D} \rightarrow \mathcal{C}$ is a functor, then it induces a new functor $F(\tau)$ : $F(\mathcal{D}) \rightarrow F(\mathcal{C})$.

Proof. For each $[\alpha] \in \mathrm{Ob} F(\mathcal{D})$ we assign to it $[\tau(\alpha)] \in \mathrm{Ob} F(\mathcal{C})$, while for each morphism $(u, v):[\alpha] \rightarrow[\beta]$ we have $(\tau(u), \tau(v)):[\tau(\alpha)] \rightarrow[\tau(\beta)]$, a morphism in $F(\mathcal{C})$. One can verify that these assignments define a functor, written as $F(\tau)$.

\section{BeCKer-Gottlleb transfer}

In this article we will focus on the functor $\pi: G \propto \mathcal{P} \rightarrow G$. The accompanying restriction $\operatorname{Res}_{\pi}$ and its left adjoint $L K_{\pi}$ will be our main tools. For instance, if $M$ is a $k G$-module, then $\operatorname{Res}_{\pi} M$ becomes a $k(G \propto \mathcal{P})$-module. Since this kind of module is of particular importance in the present article, we shall denote it as $\kappa_{M}=\operatorname{Res}_{\pi} M \in k(G \propto \mathcal{P})$-mod, following [11]. As a functor $\kappa_{M}$ takes constant value $M$ at each object of $G \propto \mathcal{P}$, but the $k(G \propto \mathcal{P})$-action on it is not trivial in general. However, the $k \mathcal{P}$-action on $\kappa_{M}$ is trivial because each morphism in $\mathcal{P}$ is 
given by the identity element $e \in G$. In the end, if $k$ is the trivial $k G$-module, $\kappa_{k}$ is identified with the trivial $k(G \propto \mathcal{P})$-module $\underline{k}$.

As a convention, throughout this article, the $k G$-modules are usually written as $M, N$, etc., while the modules of a (non-group) category algebra $k \mathcal{C}$ are denoted by $\mathfrak{M}, \mathfrak{N}$, etc., except those special modules, namely $\underline{k}$ and $\kappa_{M}$, which we have just mentioned.

3.1. Modules constructed over categories. Suppose $\mathcal{P}$ is a $G$-poset and $\pi$ is the natural functor from the transporter category $G \propto \mathcal{P}$ to $G$, regarded as a category with one object $\bullet$. The fibre of the functor $\pi\left[10\right.$, i.e. $\pi^{-1}(\bullet)$, is exactly $\mathcal{P}$. Also by [10], the overcategory $\pi / \bullet$ provides the fibre of the map $B \pi$ in the sense that the sequence of categories

$$
\pi / \bullet \rightarrow G \propto \mathcal{P} \rightarrow G
$$

corresponds to the fibration

$$
\mathcal{B}(\pi / \bullet) \rightarrow B(G \propto \mathcal{P}) \rightarrow B G .
$$

It leads to an action of $G=\pi_{1}(B G)$ on $\mathcal{B}(\pi / \bullet)$, which is realized by a $G$-action on the category $\pi / \bullet$, defined shortly after Proposition 3.1. We shall see that there exists an inclusion $\mathcal{P} \rightarrow \pi / \bullet$, although not a $G$-functor, inducing a category equivalence.

The objects of $\pi / \bullet$ are of the form $(x, h)$, in which $x \in \mathrm{Ob}(G \propto \mathcal{P})=\mathrm{Ob} \mathcal{P}$ and $h \in G$. A morphism from $(x, h)$ to $\left(x^{\prime}, h^{\prime}\right)$ is a morphism $\left(g, g x \leq x^{\prime}\right) \in \operatorname{Mor}(G \propto \mathcal{P})$ such that $h^{\prime} g=h$. It implies that each object $(x, h) \cong\left(x^{\prime}, h^{\prime}\right)$ if and only if $x^{\prime}=g x$ and $h^{\prime}=h g^{-1}$ for some $g \in G$. Indeed the objects isomorphic to $(x, h)$ are $\left\{\left(g x, h g^{-1}\right) \mid g \in G\right\}$. Particularly, $(x, h) \cong(h x, e)$ for the identity $e \in G$.

Proposition 3.1. The category $\pi / \bullet$ is isomorphic to $\left(\operatorname{Id}_{G} / \bullet\right) \times \mathcal{P}$.

Proof. We establish an isomorphism $\phi:\left(\operatorname{Id}_{G} / \bullet\right) \times \mathcal{P} \rightarrow \pi / \bullet$ as follows. The objects of $\left(\operatorname{Id}_{G} / \bullet\right) \times \mathcal{P}$ are $\{(g, x) \mid g \in G, x \in \mathrm{Ob} \mathcal{P}\}$. We define $\phi((g, x))=\left(g x, g^{-1}\right) \in$ $\mathrm{Ob}(\pi / \bullet)$. For a morphism $\left(g_{2} g_{1}^{-1}, x_{1} \leq x_{2}\right):\left(g_{1}, x_{1}\right) \rightarrow\left(g_{2}, x_{2}\right)$ we put

$$
\phi\left(\left(g_{2} g_{1}^{-1}, x_{1} \leq x_{2}\right)\right):=\left(g_{2} g_{1}^{-1},\left(g_{2} g_{1}^{-1}\right)\left(g_{1} x_{1}\right) \leq g_{2} x_{2}\right),
$$

a morphism from $\left(g_{1} x_{1}, g_{1}^{-1}\right)$ to $\left(g_{2} x_{2}, g_{2}^{-1}\right)$. The inverse $\phi^{-1}: \pi / \bullet \rightarrow\left(\operatorname{Id}_{G} / \bullet\right) \times \mathcal{P}$ is given by $\phi^{-1}((y, h)):=\left(h^{-1}, h y\right)$ and $\phi^{-1}\left(\left(h_{2}^{-1} h_{1}, h_{2}^{-1} h_{1} x_{1} \leq x_{2}\right)\right):=\left(h_{2}^{-1} h_{1}, h_{1} x_{1}\right.$ $\left.\leq h_{2} x_{2}\right)$.

The $G$-action on $\pi / \bullet$ can be explicitly described. For any object $(x, h) \in \mathrm{Ob} \pi / \bullet$ and $u \in G$, we have $u \circ(x, h)=\left(u x, h u^{-1}\right)$, and for any morphism $(g, g x \leq$ $\left.x^{\prime}\right):(x, h) \rightarrow\left(x^{\prime}, h^{\prime}\right)$ we have $u \circ\left(g, g x \leq x^{\prime}\right)=\left(u g u^{-1},\left(u g u^{-1}\right) u x \leq u x^{\prime}\right)$ : $\left(u x, h u^{-1}\right) \rightarrow\left(u x^{\prime}, h^{\prime} u^{-1}\right)$.

The proposition actually implies that $\mathcal{P}$ and $\pi / \bullet$ are equivalent. We will write the functors that induce the equivalence below. Part (1) of the following corollary was discussed and used in [15]. Since it is a direct consequence of the preceding proposition, for completion we record it here.

Corollary 3.2. (1) There exists a natural embedding $\mathcal{P} \hookrightarrow \pi / \bullet$ making $\mathcal{P}$ a skeleton of $\pi / \bullet$. Consequently $L K_{\pi} \mathfrak{M} \cong \lim _{\mathcal{P}} \mathfrak{M}$ and $R K_{\pi} \mathfrak{M} \cong \lim _{\mathcal{P}} \mathfrak{M}$ for any $\mathfrak{M} \in k(G \propto \mathcal{P})$-mod.

(2) The natural functor $\pi / \bullet \rightarrow \mathcal{P}$ is a $G$-functor. 
Proof. The natural functor $\mathcal{P} \rightarrow \pi / \bullet$, given by $x \mapsto(x, e)$ and $x \leq y \mapsto(e, x \leq y)$, is an embedding, sending $\mathcal{P}$ to a skeleton of $\pi / \bullet$. It is straightforward to check that there is a natural surjective functor $\pi / \bullet \rightarrow \mathcal{P}$, induced by $(x, h) \mapsto h x$, which is a quasi-inverse to the previous embedding. In fact, these two functors provide an equivalence between $\pi / \bullet$ and $\mathcal{P}$. The existence of an equivalence between these two categories forces $\lim _{\pi / \bullet} \mathfrak{M} \cong \lim _{\mathcal{P}} \mathfrak{M}$ and $\lim _{\pi / \bullet} \mathfrak{M} \cong \varliminf_{\mathcal{P}} \mathfrak{M}$.

It follows from our description of the $G$-action on $\pi / \bullet$ that the natural functor $\pi / \bullet \rightarrow \mathcal{P}$ is a $G$-functor.

The embedding $\mathcal{P} \hookrightarrow \pi / \bullet$ is not a $G$-functor, and one reason is that an object $x \in \mathrm{Ob} \mathcal{P}$ is mapped isomorphically to $u x \in \mathrm{Ob} \mathcal{P}$ for any $u \in G$, while its image $(x, e) \in \mathrm{Ob}(\pi / \bullet)$ must be sent to $\left(u x, u^{-1}\right) \in \mathrm{Ob}(\pi / \bullet)$, not $(u x, e)$.

By Proposition 3.1, if $\mathcal{P}=G / G=\bullet$, then $\pi_{G / G}=\operatorname{Id}_{G}$ and $\pi_{G / G} / \bullet=\operatorname{Id}_{G} / \bullet$ is the Cayley graph, giving rise to the total space $E G$ whose complex is the bar resolution $\mathcal{B}_{*}^{G}$. More generally, for $\mathcal{P}=G / H$, we have an isomorphism of complexes of $k G$-modules $\mathbb{C}_{*}(\pi / \bullet) \cong \mathcal{B}_{*}^{G} \otimes k(G / H)$, which provide a projective resolution of $k(G / H)=\mathbb{C}_{0}(G / H)=\mathbb{C}_{*}(G / H)$ (a stalk complex).

Corollary 3.3. The complex $L K_{\pi} \mathcal{B}_{*}^{G \propto \mathcal{P}} \cong \mathbb{C}_{*}(\pi / \bullet) \simeq \mathcal{B}_{*}^{G} \otimes \mathbb{C}_{*}(\mathcal{P})$ is a projective resolution of the finite complex of $k G$-modules $\mathbb{C}_{*}(\mathcal{P})$.

Proof. Since $L K_{\pi}$ preserves projectives, $L K_{\pi} \mathcal{B}_{*}^{G \propto \mathcal{P}} \cong \mathbb{C}_{*}(\pi / \bullet)$ is a complex of projective $k G$-modules. The existing $G$-functor $\pi / \bullet \rightarrow \mathcal{P}$ gives rise to a chain map of complexes of $k G$-modules $L K_{\pi} \mathcal{B}_{*}^{G \propto \mathcal{P}} \cong \mathbb{C}_{*}(\pi / \bullet) \simeq \mathcal{B}_{*}^{G} \otimes \mathbb{C}_{*}(\mathcal{P}) \rightarrow \mathbb{C}_{*}(\mathcal{P})$. However, since the $G$-functor is a category equivalence, it induces an isomorphism between the homology of complexes.

Corollary 3.4. Suppose $M \in k G$-mod and $\mathfrak{N} \in k(G \propto \mathcal{P})$-mod. Then $L K_{\pi}\left(\kappa_{M} \hat{\otimes} \mathfrak{N}\right)$ $\cong M \otimes L K_{\pi} \mathfrak{N}$ and $R K_{\pi}\left(\kappa_{M} \hat{\otimes} \mathfrak{N}\right) \cong M \otimes R K_{\pi} \mathfrak{N}$ as $k G$-modules. In particular, $L K_{\pi}\left(\kappa_{M}\right) \cong M \otimes L K_{\pi} \underline{k}$ and $R K_{\pi}\left(\kappa_{M}\right) \cong M \otimes R K_{\pi} \underline{k}$, where $L K_{\pi} \underline{k} \cong \mathrm{H}_{0}(B \mathcal{P}, k) \cong$ $\mathrm{H}^{0}(B \mathcal{P}, k) \cong R K_{\pi} \underline{k}$ of dimension equal to the number of connected components of $\mathcal{P}$.

Proof. For the left Kan extension we have

$$
L K_{\pi}\left(\kappa_{M} \hat{\otimes} \mathfrak{N}\right) \cong \lim _{\mathcal{P}}\left(\kappa_{M} \hat{\otimes} \mathfrak{N}\right) \cong M \otimes \lim _{\mathcal{P}} \mathfrak{N} \cong M \otimes L K_{\pi} \mathfrak{N} .
$$

The second isomorphism is true because $\kappa_{M}$ as a $k \mathcal{P}$-module admits trivial action. The statement for the right Kan extension is similar.

The above corollary implies that $L K_{\pi} \kappa_{M} \cong R K_{\pi} \kappa_{M}$, suggesting the existence of a transfer map, which we will construct later on.

The next result is needed to construct transfer maps. It is [16, Theorem 4.1].

Theorem 3.5. Let $\mathfrak{P} \in k(G \propto \mathcal{P})$-mod be a projective module and $\kappa_{M}=\operatorname{Res}_{\pi} M$ for some $M \in k G$-mod. Then $\mathfrak{P} \hat{\otimes} \kappa_{M}$ is a projective $k(G \propto \mathcal{P})$-module. Consequently, $\mathcal{B}_{*}^{G \propto \mathcal{P}} \hat{\otimes} \kappa_{M} \rightarrow \underline{k} \hat{\otimes} \kappa_{M}=\kappa_{M} \rightarrow 0$ is a projective resolution.

Assume $\mathbb{C}_{*}$ is a complex of $k G$-modules. Then we naturally obtain a complex of $k(G \propto \mathcal{P})$-modules via restriction, written as $\kappa_{\mathbb{C}_{*}}$. For any $M \in k G$-mod, there is naturally a chain map, unique up to homotopy, as follows:

$$
\Pi_{M}:\left\{\mathcal{B}_{*}^{G \propto \mathcal{P}} \hat{\otimes} \kappa_{M} \rightarrow \kappa_{M} \rightarrow 0\right\} \rightarrow\left\{\kappa_{\mathcal{B}_{*}^{G}} \hat{\otimes} \kappa_{M}=\kappa_{\mathcal{B}_{*}^{G} \otimes M} \rightarrow \kappa_{M} \rightarrow 0\right\} .
$$


In the proof of Theorem 3.8, for simplicity, we shall also use $\Pi_{M}$ to denote its brutal truncation

$$
\Pi_{M}:\left\{\mathcal{B}_{*}^{G \propto \mathcal{P}} \hat{\otimes} \kappa_{M}\right\} \rightarrow\left\{\kappa_{\mathcal{B}_{*}^{G}} \hat{\otimes} \kappa_{M}=\kappa_{\mathcal{B}_{*}^{G} \otimes M}\right\} .
$$

Remark 3.6. Using Dan Swenson's definition [12] (see also [15]) of the internal hom, one verifies that

$$
\kappa_{\operatorname{Hom}_{k}(M, N)} \cong \mathcal{H o m}\left(\kappa_{M}, \kappa_{N}\right) .
$$

Consequently, Theorem 3.5 implies

$$
\operatorname{Ext}_{k(G \propto \mathcal{P})}^{*}\left(\underline{k}, \kappa_{\operatorname{Hom}_{k}(M, N)}\right) \cong \operatorname{Ext}_{k(G \propto \mathcal{P})}^{*}\left(\underline{k}, \mathcal{H o m}\left(\kappa_{M}, \kappa_{N}\right)\right) \cong \operatorname{Ext}_{k(G \propto \mathcal{P})}^{*}\left(\kappa_{M}, \kappa_{N}\right) \text {. }
$$

3.2. Equivariant cohomology. Before moving to the next application, we record a connection between cohomology of transporter categories and equivariant cohomology, which is perhaps known to the experts.

Proposition 3.7. The left Kan extension induces an isomorphism

$$
\lambda_{M}: \operatorname{Ext}_{k(G \propto \mathcal{P})}^{*}\left(\underline{k}, \kappa_{M}\right) \cong \mathrm{H}_{G}^{*}(B \mathcal{P}, M),
$$

where $\mathrm{H}_{G}^{*}(B \mathcal{P}, M)$ is the equivariant cohomology group for some $M \in k G$-mod.

Proof. Take the bar resolution $\mathcal{B}_{*}^{G \propto \mathcal{P}} \rightarrow \underline{k} \rightarrow 0$ and consider the complex $\operatorname{Hom}_{k(G \propto \mathcal{P})}\left(\mathcal{B}_{*}^{G \propto \mathcal{P}}, \kappa_{M}\right)$. The left Kan extension induces a chain map

$$
\operatorname{Hom}_{k(G \propto \mathcal{P})}\left(\mathcal{B}_{*}^{G \propto \mathcal{P}}, \kappa_{M}\right) \cong \operatorname{Hom}_{k G}\left(L K_{\pi} \mathcal{B}_{*}^{G \propto \mathcal{P}}, M\right) \simeq \operatorname{Hom}_{k G}\left(\mathcal{B}_{*}^{G} \otimes \mathbb{C}_{*}(\mathcal{P}), M\right),
$$

but the rightmost term is

$$
\operatorname{Hom}_{k G}\left(\mathcal{B}_{*}^{G} \otimes \mathbb{C}_{*}(\mathcal{P}), M\right) \cong \operatorname{Hom}_{k G}\left(\mathcal{B}_{*}^{G}, \operatorname{Hom}_{k}\left(\mathbb{C}_{*}(\mathcal{P}), M\right)\right),
$$

which gives rise to $\mathrm{H}_{G}^{*}(B \mathcal{P}, M)$.

In light of the above proposition, we may introduce the Tate cohomology of transporter categories as Tate equivariant cohomology. With Remark 3.6 in mind, one can further define negative degree Ext groups as $\operatorname{Ext}_{k(G \propto \mathcal{P})}^{*}\left(\kappa_{M}, \kappa_{N}\right)$.

3.3. Construction of the transfer map. Let $\tau: \mathcal{D} \rightarrow \mathcal{C}$ be a functor between small categories. There is always a restriction $\operatorname{res}_{\tau}: \mathrm{H}^{*}(\mathcal{C} ; \underline{k}) \rightarrow \mathrm{H}^{*}(\mathcal{D} ; \underline{k})$. However, usually one cannot construct a map in the opposite direction, unless the two Kan extensions are connected by a natural transformation. In this section, based on our knowledge about representations of $k(G \propto \mathcal{P})$, we establish the Becker-Gottlieb transfer map with respect to $\pi_{\mathcal{P}}: G \propto \mathcal{P} \rightarrow G$. Here we provide an algebraic alternative to the construction of Becker-Gottlieb [1], and the core idea is taken from Dwyer-Wilkerson [6, 9.13]. Essentially our construction incorporates [6, 9.13] in an entirely representation-theoretic setting. The upshot is that our construction is analogous to the classical situation; see for instance [2, 3.6.17]. Keep in mind that $\operatorname{Res}_{\pi}$ and $L K_{\pi}$ generalize $\downarrow_{H}^{G}$ and $\uparrow_{H}^{G}$, respectively, used in group cohomology.

Theorem 3.8. Suppose $\operatorname{Res}_{\pi}: k G$-mod $\rightarrow k(G \propto \mathcal{P})$-mod is the restriction along $\pi$ and write $\kappa_{M}=\operatorname{Res}_{\pi} M$ for any $M \in k G$-mod. Then we have the following two maps, restriction and transfer,

$$
\operatorname{Ext}_{k G}^{*}(M, N) \stackrel{\mathrm{res}_{\mathcal{P}}}{\rightarrow} \operatorname{Ext}_{k(G \propto \mathcal{P})}^{*}\left(\kappa_{M}, \kappa_{N}\right) \stackrel{\operatorname{tr}_{\mathcal{P}}}{\rightarrow} \operatorname{Ext}_{k G}^{*}(M, N),
$$

which compose to $\chi(\mathcal{P} ; k) \cdot 1$, multiplication by the Euler characteristic of (the order complex of $) \mathcal{P}$. When $\mathcal{P}=G / H$ for some subgroup $H, \operatorname{tr}_{G / H}$ is the usually transfer map in group cohomology. 
Proof. We shall construct these two maps. Then in the sequel one can deduce the statement on their composite.

Suppose $\mathcal{B}_{*}^{G}$ and $\mathcal{B}_{*}^{G \propto \mathcal{P}}$ are the bar resolutions of $k \in k G$-mod and $\underline{k} \in k(G \propto$ $\mathcal{P})$-mod, respectively. Then $\mathcal{B}_{*}^{G} \otimes M$ is a projective resolution of a fixed $M \in$ $k G$-mod. For another $k G$-module $N$, the cochain complex $\operatorname{Hom}_{k G}\left(\mathcal{B}_{*}^{G} \otimes M, N\right)$ computes $\operatorname{Ext}_{k G}^{*}(M, N)$. The exact functor $\operatorname{Res}_{\pi}$ sends this cochain complex to $\operatorname{Hom}_{k(G \propto \mathcal{P})}\left(\kappa_{\mathcal{B}_{*}^{G} \otimes M}, \kappa_{N}\right)$. From Theorem 3.5 we obtain the composite of two chain maps, unique up to chain homotopy,

$$
\operatorname{Hom}_{k G}\left(\mathcal{B}_{*}^{G} \otimes M, N\right) \stackrel{\text { Res }^{\pi}}{\rightarrow} \operatorname{Hom}_{k(G \propto \mathcal{P})}\left(\kappa_{\mathcal{B}_{*}^{G} \otimes M}, \kappa_{N}\right) \stackrel{\Pi_{M}^{*}}{\rightarrow} \operatorname{Hom}_{k(G \propto \mathcal{P})}\left(\mathcal{B}_{*}^{G \propto \mathcal{P}} \hat{\otimes} \kappa_{M}, \kappa_{N}\right) .
$$

These seemingly abstract maps can be spelled out explicitly, but we will leave it to the interested reader. The composite $\Pi_{M}^{*} \circ \operatorname{Res}_{\pi}$ induces a map on cohomology, which we call the restriction:

$$
\operatorname{res}_{\mathcal{P}}: \operatorname{Ext}_{k G}^{*}(M, N) \rightarrow \operatorname{Ext}_{k(G \propto \mathcal{P})}^{*}\left(\kappa_{M}, \kappa_{N}\right) .
$$

It is helpful to have a different characterization of the restriction. In order to do so we use a series of obvious isomorphisms to rewrite the previously mentioned complex $\operatorname{Hom}_{k(G \propto \mathcal{P})}\left(\mathcal{B}_{*}^{G \propto \mathcal{P}} \hat{\otimes} \kappa_{M}, \kappa_{N}\right)$. Firstly, by adjunction, it is isomorphic to

$$
\operatorname{Hom}_{k G}\left(L K_{\pi}\left(\mathcal{B}_{*}^{G \propto \mathcal{P}} \hat{\otimes} \kappa_{M}\right), N\right) .
$$

Here $\left(\Pi_{M}^{*} \circ \operatorname{Res}_{\pi}\right) \alpha$ is mapped to $\left(\Lambda_{N} \circ L K_{\pi}\right)\left(\Pi_{M}^{*} \circ \operatorname{Res}_{\pi} \alpha\right)$, where $\Lambda_{N}: L K_{\pi} \kappa_{N} \rightarrow N$, determined by the counit of adjunction, is isomorphic to $\bar{\epsilon} \otimes \operatorname{Id}_{N}$. The natural map $\bar{\epsilon}: \mathrm{H}_{0}(\mathcal{P}) \rightarrow k$ is induced by the augmentation map $\epsilon: \mathbb{C}_{0}(\mathcal{P}) \rightarrow k$ (or rather $\mathcal{P} \rightarrow \bullet$ ) by Corollary 3.4. Secondly, from the same corollary our cochain complex is canonically isomorphic to

$$
\operatorname{Hom}_{k G}\left(L K_{\pi}\left(\mathcal{B}_{*}^{G \propto \mathcal{P}}\right) \otimes M, N\right) .
$$

Thirdly, by Corollary 3.3 where $L K_{\pi} \mathcal{B}_{*}^{G \propto \mathcal{P}} \cong \mathcal{B}_{*}^{G} \otimes \mathbb{C}_{*}(\mathcal{P})$, the above complex is

$$
\begin{aligned}
\operatorname{Hom}_{k G}\left(L K_{\pi}\left(\mathcal{B}_{*}^{G \propto \mathcal{P}}\right) \otimes M, N\right) & \simeq \operatorname{Hom}_{k G}\left(\mathcal{B}_{*}^{G} \otimes \mathbb{C}_{*}(\mathcal{P}) \otimes M, N\right) \\
& \cong \operatorname{Hom}_{k G}\left(\mathcal{B}_{*}^{G} \otimes M \otimes \mathbb{C}_{*}(\mathcal{P}), N\right) .
\end{aligned}
$$

The observations imply that $\operatorname{res}_{\mathcal{P}}: \operatorname{Ext}_{k G}^{*}(M, N) \rightarrow \operatorname{Ext}_{k(G \propto \mathcal{P})}^{*}\left(\kappa_{M}, \kappa_{N}\right)$ is the same as the map induced by the following chain map:

$$
\operatorname{Hom}_{k G}\left(\mathcal{B}_{*}^{G} \otimes M, N\right) \rightarrow \operatorname{Hom}_{k G}\left(\mathcal{B}_{*}^{G} \otimes M \otimes \mathbb{C}_{*}(\mathcal{P}), N\right),
$$

which is given by the augmentation $\epsilon: \mathbb{C}_{*}(\mathcal{P}) \rightarrow k$. Now we are ready to build the transfer map. From the cochain complex $\operatorname{Hom}_{k G}\left(\mathcal{B}_{*}^{G} \otimes M \otimes \mathbb{C}_{*}(\mathcal{P}), N\right)$ we continue to establish a map which leads back to group cohomology:

$$
\Theta^{*}: \operatorname{Hom}_{k G}\left(\mathcal{B}_{*}^{G} \otimes M \otimes \mathbb{C}_{*}(\mathcal{P}), N\right) \rightarrow \operatorname{Hom}_{k G}\left(\mathcal{B}_{*}^{G} \otimes M, N\right) .
$$

The map $\Theta^{*}$ is induced by the chain map $\Theta: k \rightarrow \mathbb{C}_{*}(\mathcal{P})$ of Dwyer-Wilkerson [6. 9.13], as the composite of

$$
\begin{aligned}
& k \stackrel{a \mapsto a \cdot \operatorname{Id}}{\rightarrow} \operatorname{Hom}_{k}\left(\mathbb{C}_{*}(\mathcal{P}), \mathbb{C}_{*}(\mathcal{P})\right) \stackrel{\cong}{\rightarrow} \mathbb{C}_{*}(\mathcal{P})^{\wedge} \otimes \mathbb{C}_{*}(\mathcal{P}) \\
& \stackrel{\operatorname{Id} \otimes \Delta}{\rightarrow} \mathbb{C}_{*}(\mathcal{P})^{\wedge} \otimes \mathbb{C}_{*}(\mathcal{P}) \otimes \mathbb{C}_{*}(\mathcal{P}) \stackrel{e v \otimes}{\rightarrow} \mathbb{C}_{*}(\mathcal{P}) .
\end{aligned}
$$


Here $\mathbb{C}_{*}(\mathcal{P})^{\wedge}=\operatorname{Hom}_{k}\left(\mathbb{C}_{*}(\mathcal{P}), k\right)$, non-positively graded, is the $k$-dual of $\mathbb{C}_{*}(\mathcal{P})$, $\Delta$ is the diagonal map and $e v$ is the evaluation map. The composite of

$\operatorname{Hom}_{k(G \propto \mathcal{P})}\left(\mathcal{B}_{*}^{G \propto \mathcal{P}} \hat{\otimes} \kappa_{M}, \kappa_{N}\right) \stackrel{\cong}{\rightrightarrows} \operatorname{Hom}_{k G}\left(L K_{\pi}\left(\mathcal{B}_{*}^{G \propto \mathcal{P}} \hat{\otimes} \kappa_{M}\right), N\right) \stackrel{\Theta^{*}}{\rightarrow} \operatorname{Hom}_{k G}\left(\mathcal{B}_{*}^{G} \otimes M, N\right)$ defines a map

$$
\operatorname{tr}_{\mathcal{P}}: \operatorname{Ext}_{k(G \propto \mathcal{P})}^{*}\left(\kappa_{M}, \kappa_{N}\right) \rightarrow \operatorname{Ext}_{k G}^{*}(M, N),
$$

which is called the transfer.

In fact we have the following commutative diagram of cochain complexes:

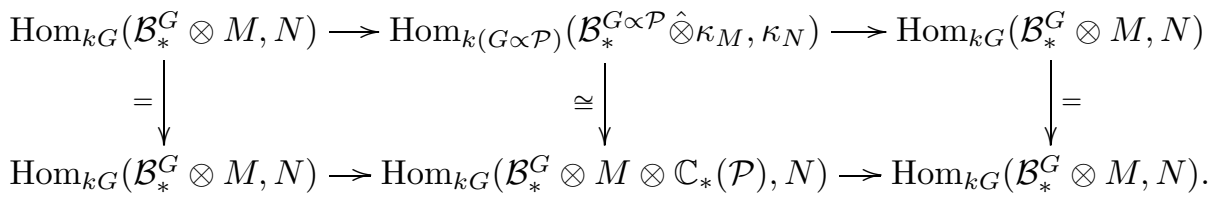

Upon passing to cohomology, both rows give rise to

$$
\operatorname{Ext}_{k G}^{*}(M, N) \stackrel{\text { res } \mathcal{P}}{\rightarrow} \operatorname{Ext}_{k(G \propto \mathcal{P})}^{*}\left(\kappa_{M}, \kappa_{N}\right) \stackrel{\mathrm{tr}_{\mathcal{P}}}{\rightarrow} \operatorname{Ext}_{k G}^{*}(M, N) .
$$

In the end we prove that the composite $\operatorname{res}_{\mathcal{P}} \operatorname{otr}_{\mathcal{P}}=\chi(\mathcal{P}) \cdot 1$. We directly compute the composite

$$
k \rightarrow \mathbb{C}_{*}(\mathcal{P}) \rightarrow k
$$

and show that it is a scalar multiplication by $\chi(\mathcal{P})$. Write the natural basis of $\mathbb{C}_{n}(\mathcal{P})$ as $\left\{c_{n}^{i}\right\}_{i=1}^{d_{n}}$ for $d_{n}=\operatorname{dim}_{k} \mathbb{C}_{n}(\mathcal{P})$ (see Section 2.2). We have

$$
\begin{aligned}
1 & \mapsto \operatorname{Id}_{\mathbb{C}_{*}(\mathcal{P})} \\
& \mapsto \sum_{n=0}^{d}(-1)^{n}\left\{\sum_{i=1}^{d_{n}}\left(c_{n}^{i}\right)^{\wedge} \otimes c_{n}^{i}\right\} \\
& \mapsto \sum_{n=0}^{d}(-1)^{n}\left\{\sum_{i=0}^{d_{n}}\left(c_{n}^{i}\right)^{\wedge} \otimes\left[c_{n}^{i} \otimes t\left(c_{n}^{i}\right)\right]\right\} \\
& =\sum_{n=0}^{d}(-1)^{n}\left\{\sum_{i=1}^{d_{n}}\left[\left(c_{n}^{i}\right)^{\wedge} \otimes c_{n}^{i}\right] \otimes t\left(c_{n}^{i}\right)\right\} \\
& \mapsto \sum_{n=0}^{d}(-1)^{n}\left\{\sum_{i=1}^{d_{n}} t\left(c_{n}^{i}\right)\right\} \\
& \mapsto \sum_{n=0}^{d}(-1)^{n} d_{n} \\
& =\chi(\mathcal{P}) .
\end{aligned}
$$

Here $t\left(c_{n}^{i}\right) \in \mathbb{C}_{0}(\mathcal{P})$ denotes the last object, i.e. the target, of the $n$-chain of morphisms $c_{n}^{i} \in \mathbb{C}_{n}(\mathcal{P})$.

It is well known that, in the cohomology of algebras, if $\rho: A \rightarrow B$ is an algebra homomorphism and $V, W$ are two $B$-modules, then one can construct a natural map $\operatorname{res}_{\rho}: \operatorname{Ext}_{B}^{*}(V, W) \rightarrow \operatorname{Ext}_{A}^{*}\left(\operatorname{Res}_{\rho} V, \operatorname{Res}_{\rho} W\right)$. This cannot be used in our situation because the functor $\pi: G \propto \mathcal{P} \rightarrow G$ does not induce a homomorphism between their category algebras. 
Remark 3.9. In fact, by Remark 3.6, the above restriction and transfer coincide with

$$
\operatorname{Ext}_{k G}^{*}\left(k, \operatorname{Hom}_{k}(M, N)\right) \stackrel{\mathrm{res}_{\mathcal{P}}}{\rightarrow} \operatorname{Ext}_{k(G \propto \mathcal{P})}^{*}\left(\underline{k}, \kappa_{\operatorname{Hom}_{k}(M, N)}\right) \stackrel{\operatorname{tr}_{\mathcal{P}}}{\rightarrow} \operatorname{Ext}_{k G}^{*}\left(k, \operatorname{Hom}_{k}(M, N)\right) .
$$

When $M=N=k$, our construction is exactly the Becker-Gottlieb transfer ([1], [6]), because $\operatorname{Ext}_{k(G \propto \mathcal{P})}^{*}\left(\underline{k}, \kappa_{M}\right) \cong \mathrm{H}_{G}^{*}(B \mathcal{P}, M)$.

We emphasize that if either $M \in k G$-mod is not acted trivially by $k G$ or if $G$ sends a connected component of $\mathcal{P}$ to a different one, then the constantly valued $\kappa_{M} \in k(G \propto \mathcal{P})$-mod is not truly constant since $\mathrm{H}^{0}\left(G \propto \mathcal{P} ; \kappa_{M}\right) \cong \lim _{G \propto \mathcal{P}} \kappa_{M} \cong$ $\lim _{G} \lim _{\mathcal{P}} \kappa_{M} \cong\left(M \otimes \mathrm{H}^{0}(\mathcal{P})\right)^{G}$.

\section{Transfer FOR Hochschild COHOMOLOGY}

Using exactly the same method we can build a transfer map between Hochschild cohomology. Consider the following commutative diagram of functors:

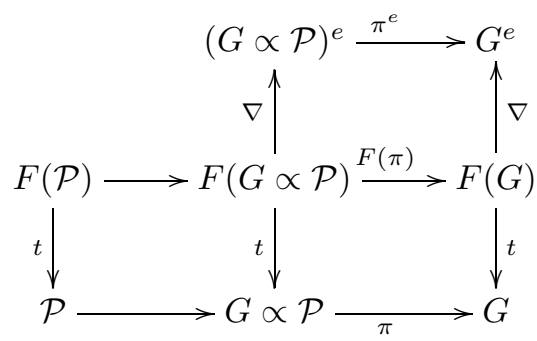

Recall that in the last section, based on the bottom row, we were able to establish a transfer map between ordinary cohomology. Since the three target functors all induce homotopy equivalences, the middle row may as well give a transfer map $\operatorname{Ext}_{k F(G \propto \mathcal{P})}^{*}(-,-) \rightarrow \operatorname{Ext}_{k F(G)}^{*}(-,-)$, for suitable modules. Since furthermore the upper right square demonstrates connections between the ordinary cohomology over $k F(G \propto \mathcal{P})$ and $k F(G)$ with the Hochschild cohomology of $k F(G \propto \mathcal{P})$ and $k F(G)$, respectively, we automatically wonder if there would be a transfer between the Hochschild cohomology of $k(G \propto \mathcal{P})$ and $k G$. The answer is yes, and a predecessor of this construction is due to Linckelmann [8]. For $G$ a group and $H$ a subgroup, he developed a method over symmetric algebras and defined a transfer map from $\mathrm{HH}^{*}(k H)$ to $\mathrm{HH}^{*}(k G)$. Recall that $H$ can be recovered as the transporter category $G \propto(G / H)$, and our construction generalizes his.

Lemma 4.1. Let $k G \in k G^{e}$-mod and $k(G \propto \mathcal{P}) \in k(G \propto \mathcal{P})^{e}$-mod. Then the $k F(G \propto \mathcal{P})$-module $\operatorname{Res}_{F(\pi)} \operatorname{Res}_{\nabla} k G$ contains $\operatorname{Res}_{\nabla} k(G \propto \mathcal{P})$ as a submodule.

Proof. Let $(x, y) \in \mathrm{Ob}(G \propto \mathcal{P})^{e}$. Then

$$
\begin{aligned}
k(G \propto \mathcal{P})(x, y) & =k \operatorname{Hom}_{G \propto \mathcal{P}}(y, x) \\
& =k\{(h, h y \leq x) \mid h \in G\} \\
& \subset k G \\
& =\left(\operatorname{Res}_{\pi^{e}} k G\right)(x, y) .
\end{aligned}
$$

From here we can verify that $k(G \propto \mathcal{P})$ is a $k(G \propto \mathcal{P})^{e}$-submodule of $\operatorname{Res}_{\pi^{e}} k G$. Hence the result follows. 
If $\mathcal{P}=G / H$, then $G \propto(G / H) \simeq H$ and $F(G \propto(G / H)) \simeq H$. The above inclusion says that the $k H$-module $k G \downarrow_{H}^{G}$ contains $k H$ as a submodule. Here $k G$ and $k H$ are acted upon by $G$ and $H$, respectively, by conjugation.

Lemma 4.2. Consider the functor $F(\pi): F(G \propto \mathcal{P}) \rightarrow F(G)$. Then for any $[g] \in \mathrm{Ob} F(G), F(\pi) /[g] \cong F(\mathcal{P})_{[g]} \times \operatorname{Id}_{F(G)} /[g]$, where $F(\mathcal{P})_{[g]}$ denotes a poset, indexed by $[g] \in \mathrm{Ob} F(G)$, which is canonically isomorphic to $F(\mathcal{P})$. Consequently,

$$
\mathbb{C}_{*}(F(\pi) /[g]) \cong \mathbb{C}_{*}\left(F(\mathcal{P})_{[g]} \times \operatorname{Id}_{F(G)} /[g]\right),
$$

as complexes of $k F(G)$-modules. Since $F(G) \simeq G$, for the identity element $e \in G$,

$$
\mathbb{C}_{*}(F(\pi) /[e]) \cong \mathbb{C}_{*}\left(F(\mathcal{P})_{[e]} \times \operatorname{Id}_{F(G)} /[e]\right) \simeq \mathbb{C}_{*}(F(\mathcal{P})) \otimes \mathcal{B}_{*}^{G}
$$

is a projective resolution of the complex of $k G$-modules $\mathbb{C}_{*}(F(\mathcal{P}))$.

Proof. Suppose $[g]$ is an object in $F(G)$. Then the overcategory $F(G) /[g]$ has objects $\left\{\left([(h, h x \leq y)],\left(h_{1}, h_{2}\right)\right)\right\}$, in which $[(h, h x \leq y)] \in \operatorname{Ob} F(G \propto \mathcal{P})$. It implies $g=h_{1} h h_{2}$. As a consequence, $h x \leq y$ is equivalent to $h_{2}^{-1} x \leq g^{-1} h_{1} y$. Morphisms $\left(\left(v_{1}, v_{1} y \leq y^{\prime}\right),\left(v_{2}, v_{2} x^{\prime} \leq x\right)\right)$ are given by

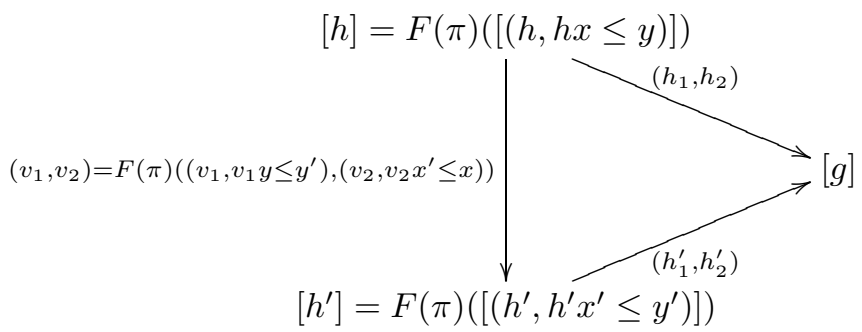

It implies various identities: $g=h_{1} h h_{2}=h_{1}^{\prime} h^{\prime} h_{2}^{\prime}, h_{1}=h_{1}^{\prime} v_{1}$ and $h_{2}=v_{2} h_{2}^{\prime}$. We define a functor $F(\pi) /[g] \rightarrow F(\mathcal{P}) \times \operatorname{Id}_{F(G)} /[g]$ such that on objects

$$
\left([(h, h x \leq y)],\left(h_{1}, h_{2}\right)\right) \mapsto\left(\left[h_{2}^{-1} x \leq g^{-1} h_{1} y\right],\left([h],\left(h_{1}, h_{2}\right)\right)\right)
$$

and on morphisms $\left(\left(v_{1}, v_{1} y \leq y^{\prime}\right),\left(v_{2}, v_{2} x^{\prime} \leq x\right)\right) \mapsto\left((e, e),\left(v_{1}, v_{2}\right)\right)$, because $v_{1} y \leq$ $y^{\prime}$ implies $g^{-1} h_{1} y \leq g^{-1} h_{1}^{\prime} y^{\prime}$ while $v_{2} x^{\prime} \leq x$ implies $h_{2}^{\prime-1} x^{\prime} \leq h_{2}^{-1} x$. The inverse of this functor is defined by $\left([x \leq y],\left([h],\left(h_{1}, h_{2}\right)\right)\right) \mapsto\left(\left[\left(h, h\left(h_{2} x\right) \leq h_{1}^{-1} g y\right)\right],\left(h_{1}, h_{2}\right)\right)$ on objects, and on morphisms it is defined by $\left((e, e),\left(v_{1}, v_{2}\right)\right) \mapsto\left(\left(v_{1}, v_{1} h_{1}^{-1} g y \leq\right.\right.$ $\left.\left.h_{1}^{\prime-1} g y^{\prime}\right),\left(v_{2}, v_{2} h_{2}^{\prime} x^{\prime} \leq h_{2} x\right)\right)$. Thus we obtain an isomorphism

$$
F(\pi) /[g] \cong F(\mathcal{P})_{[g]} \times \operatorname{Id}_{F}(G) /[g],
$$

where $F(\mathcal{P})_{[g]}$ denotes a copy of $F(\mathcal{P})$ indexed by the object $[g]$. If $\left(l_{1}, l_{2}\right):[g] \rightarrow\left[g^{\prime}\right]$ is a morphism in $F(G)$, then it induces a functor $F(\pi) /[g] \rightarrow F(\pi) /\left[g^{\prime}\right]$ given by

$$
\left([(h, h x \leq y)],\left(h_{1}, h_{2}\right)\right) \mapsto\left([(h, h x \leq y)],\left(l_{1} h_{1}, h_{2} l_{2}\right)\right)
$$

and

$$
\left(\left(v_{1}, v_{1} y \leq y^{\prime}\right),\left(v_{2}, v_{2} x^{\prime} \leq x\right)\right) \mapsto\left(\left(v_{1}, v_{1} y \leq y^{\prime}\right),\left(v_{2}, v_{2} x^{\prime} \leq x\right)\right) .
$$

Using the isomorphisms $F(\pi) /[g] \cong F(\mathcal{P})_{[g]} \times \operatorname{Id}_{F(G)} /[g]$ and $F(\pi) /\left[g^{\prime}\right] \cong F(\mathcal{P})_{\left[g^{\prime}\right]} \times$ $\operatorname{Id}_{F(G)} /\left[g^{\prime}\right]$, one can see it induces an isomorphism $F(\mathcal{P})_{[g]} \rightarrow F(\mathcal{P})_{\left[g^{\prime}\right]}$. 
Finally, since $G$ is isomorphic to the automorphism group of $[e]$ in the groupoid $F(G)$, we have an equivalence $F(G) \simeq G$. Thus $\mathbb{C}_{*}\left(F(\mathcal{P})_{[e]} \times \operatorname{Id}_{F(G)} /[e]\right) \simeq$ $\mathbb{C}_{*}(F(\mathcal{P})) \otimes \mathbb{C}_{*}\left(\operatorname{Id}_{F(G)} /[e]\right)$ is a complex of projective $k G$-modules. Furthermore, because $\mathbb{C}_{*}\left(\operatorname{Id}_{F(G)} /[e]\right)=\mathcal{B}_{*}^{F(G)}([e])$, it has to be a projective resolution of the trivial $k G$-module $k$. Hence we get the chain homotopy equivalence as stated.

This lemma allows us to describe the transfer map in Theorem 3.8 in terms of factorization categories:

$$
\operatorname{tr}_{\mathcal{P}}: \operatorname{Ext}_{k F(G \propto \mathcal{P})}^{*}(\underline{k}, \underline{k}) \rightarrow \operatorname{Ext}_{k F(G)}^{*}(\underline{k}, \underline{k}) .
$$

This makes sense because from Section 2.4, for any $\mathcal{C}$, the functor $t: F(\mathcal{C}) \rightarrow \mathcal{C}$ induces an isomorphism $\operatorname{Ext}_{k F(\mathcal{C})}^{*}(\underline{k}, \underline{k}) \cong \operatorname{Ext}_{k \mathcal{C}}^{*}(\underline{k}, \underline{k})$. Indeed by Lemma 2.5 we have a commutative diagram

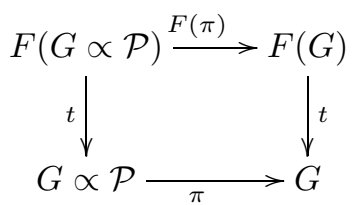

Thus we get a commutative diagram of cochain complexes

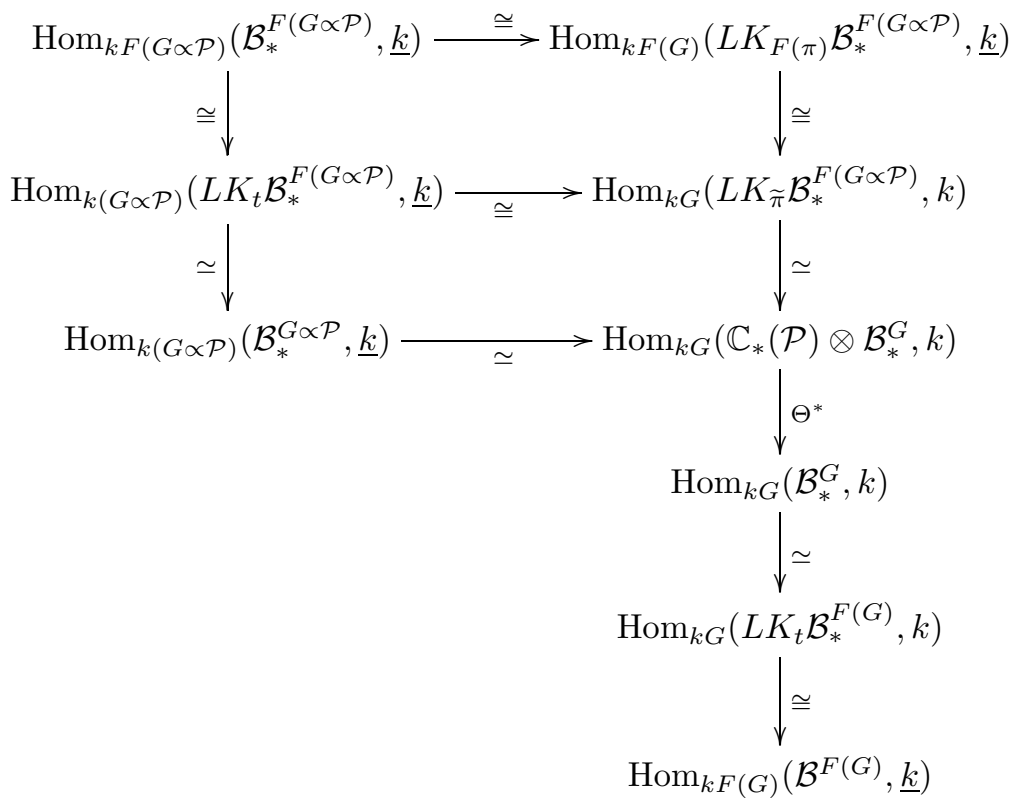

The isomorphisms are given by adjunctions and the chain homotopy equivalences are induced by changing projective resolutions. From the upper left corner to the lower right corner is the transfer that we want to describe. It factors through $\Theta^{*}$, induced by the chain map of Dwyer-Wilkerson for $M=N=k$, which gives rise to the transfer constructed in Theorem 3.8. 
Theorem 4.3. There exists a transfer map

$$
\operatorname{htr}_{\mathcal{P}}: \operatorname{Ext}_{k(G \propto \mathcal{P})^{e}}^{*}(k(G \propto \mathcal{P}), k(G \propto \mathcal{P})) \rightarrow \operatorname{Ext}_{k G^{e}}^{*}(k G, k G),
$$

which is compatible with the transfer map $\operatorname{tr}_{\mathcal{P}}$ in Theorem 3.8, in the sense that there exists a commutative diagram

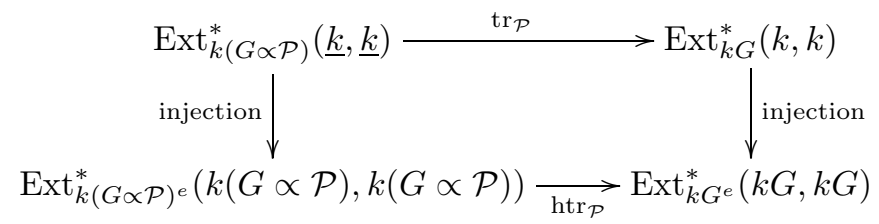

Proof. Using Lemma 4.2 we have cochain maps

$$
\begin{aligned}
\operatorname{Hom}_{k F(G \propto \mathcal{P})}\left(\mathcal{B}_{*}^{G \propto \mathcal{P}}, \operatorname{Res}_{F(\pi)} \operatorname{Res}_{\nabla} k G\right) & \cong \operatorname{Hom}_{k F(G)}\left(L K_{F(\pi)} \mathcal{B}_{*}^{G \propto \mathcal{P}}, \operatorname{Res}_{\nabla} k G\right) \\
& \cong \operatorname{Hom}_{k F(G)}\left(\mathbb{C}_{*}(F(\pi) /-), \operatorname{Res}_{\nabla} k G\right) \\
& \cong \operatorname{Hom}_{k G}\left(\mathbb{C}_{*}(F(\pi) /[e]), k G\right) \\
& \simeq \operatorname{Hom}_{k G}\left(\mathbb{C}_{*}(F(\mathcal{P})) \otimes \mathcal{B}_{*}^{G}, k G\right) \\
& \rightarrow \operatorname{Hom}_{k G}\left(\mathcal{B}_{*}^{G}, k G\right) .
\end{aligned}
$$

Here the third isomorphism is true because $F(G)$ is a groupoid whose skeleton is isomorphic to the automorphism group of $[e]$, which is exactly $G$. The module $k G$ in $\operatorname{Hom}_{k G}(-, k G)$ is acted upon by $k G$ via conjugations. The last map is induced by $k \rightarrow \mathbb{C}_{*}(F(\mathcal{P}))$, a chain map constructed in the same way as the one in the proof of Theorem 3.8, $k \rightarrow \mathbb{C}_{*}(\mathcal{P})$. Note that the functor $t: F(\mathcal{P}) \rightarrow \mathcal{P}$ induces a homotopy equivalence $B F(\mathcal{P}) \simeq B \mathcal{P}$, and thus a chain homotopy equivalence $\mathbb{C}_{*}(F(\mathcal{P})) \simeq \mathbb{C}_{*}(\mathcal{P})$.

By Lemma 4.1 we also have a chain map

$$
\operatorname{Hom}_{k F(G \propto \mathcal{P})}\left(\mathcal{B}_{*}^{G \propto \mathcal{P}}, \operatorname{Res}_{\nabla} k F(G \propto \mathcal{P})\right) \rightarrow \operatorname{Hom}_{k F(G \propto \mathcal{P})}\left(\mathcal{B}_{*}^{G \propto \mathcal{P}}, \operatorname{Res}_{F(\pi)} \operatorname{Res}_{\nabla} k G\right)
$$

induced by the inclusion $\operatorname{Res}_{\nabla} k F(G \propto \mathcal{P}) \rightarrow \operatorname{Res}_{F(\pi)} \operatorname{Res}_{\nabla} k G$. Hence altogether we obtain a chain map

$$
\operatorname{Hom}_{k F(G \propto \mathcal{P})}\left(\mathcal{B}_{*}^{G \propto \mathcal{P}}, \operatorname{Res}_{\nabla} k F(G \propto \mathcal{P})\right) \rightarrow \operatorname{Hom}_{k G}\left(\mathcal{B}_{*}^{G}, k G\right) .
$$

Passing to cohomology we get a map between Hochschild cohomology (see Section 2.4):

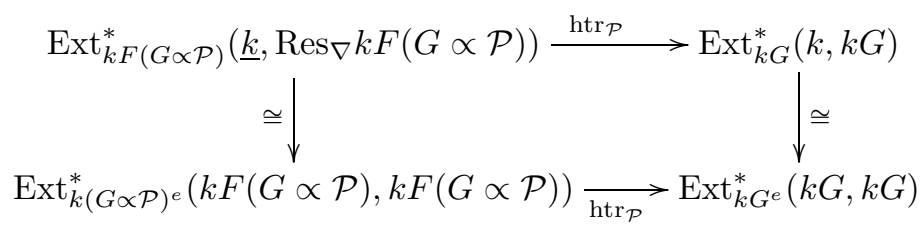

The above map deserves to be called a transfer since $\underline{k} \mid \operatorname{Res}_{\nabla} k F(G \propto \mathcal{P})$, $k \mid k G$ ( $k G$ acting on $k G$ via conjugation $c_{g}=g(-) g^{-1}$; see [3. Theorem 2.11.2]) and $\underline{k} \mid \operatorname{Res} \nabla k F(G)$. In the construction of $\operatorname{htr}_{\mathcal{P}}$ if we replace the second module 
in all $\mathrm{Hom}_{-}(-,-)$and $\operatorname{Ext}_{-}^{*}(-,-)$ by $\underline{k}$, then it is exactly the transfer created in Theorem 3.8 and reinterpreted before Theorem 4.3. In other words, we have a commutative diagram

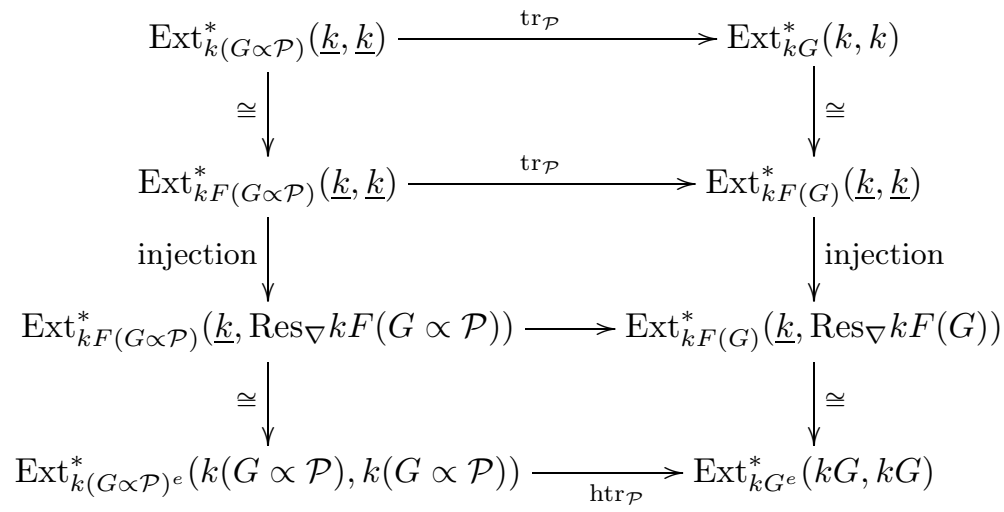

\section{REFERENCES}

[1] J. C. Becker and D. H. Gottlieb, Transfer maps for fibrations and duality, Compositio Math. 33 (1976), no. 2, 107-133. MR0436137 (55 \#9087)

[2] D. J. Benson, Representations and cohomology. I. Basic representation theory of finite groups and associative algebras, 2nd ed., Cambridge Studies in Advanced Mathematics, vol. 30, Cambridge University Press, Cambridge, 1998. MR.1644252 (99f:20001a)

[3] D. J. Benson, Representations and cohomology. II. Cohomology of groups and modules, 2nd ed., Cambridge Studies in Advanced Mathematics, vol. 31, Cambridge University Press, Cambridge, 1998. MR 1634407 (99f:20001b)

[4] W. G. Dwyer, Homology decompositions for classifying spaces of finite groups, Topology 36 (1997), no. 4, 783-804, DOI 10.1016/S0040-9383(96)00031-6. MR1432421 (97m:55016)

[5] William G. Dwyer and Hans-Werner Henn, Homotopy theoretic methods in group cohomology, Advanced Courses in Mathematics. CRM Barcelona, Birkhäuser Verlag, Basel, 2001. MR.1926776 (2003h:20093)

[6] W. G. Dwyer and C. W. Wilkerson, Homotopy fixed-point methods for Lie groups and finite loop spaces, Ann. of Math. (2) 139 (1994), no. 2, 395-442, DOI 10.2307/2946585. MR 1274096 (95e:55019)

[7] P. J. Hilton and U. Stammbach, A course in homological algebra, 2nd ed., Graduate Texts in Mathematics, vol. 4, Springer-Verlag, New York, 1997. MR.1438546 (97k:18001)

[8] Markus Linckelmann, Transfer in Hochschild cohomology of blocks of finite groups, Algebr. Represent. Theory 2 (1999), no. 2, 107-135, DOI 10.1023/A:1009979222100. MR1702272 (2000h:20024)

[9] Saunders Mac Lane, Categories for the working mathematician, 2nd ed., Graduate Texts in Mathematics, vol. 5, Springer-Verlag, New York, 1998. MR.1712872 (2001j:18001)

[10] Daniel Quillen, Higher algebraic $K$-theory. I, Algebraic $K$-theory, I: Higher $K$-theories (Proc. Conf., Battelle Memorial Inst., Seattle, Wash., 1972), Lecture Notes in Math., Vol. 341, Springer, Berlin, 1973, pp. 85-147. MR0338129 (49 \#2895)

[11] Mark A. Ronan and Stephen D. Smith, Sheaves on buildings and modular representations of Chevalley groups, J. Algebra 96 (1985), no. 2, 319-346, DOI 10.1016/0021-8693(85)90013-4. MR.810532(87h:20087)

[12] Daniel E. Swenson, The Steinberg complex of an arbitrary finite group in arbitrary positive characteristic, Thesis (Ph.D.)-University of Minnesota, 2009, ProQuest LLC, Ann Arbor, MI. MR2713697

[13] Peter Webb, An introduction to the representations and cohomology of categories, Group representation theory, EPFL Press, Lausanne, 2007, pp. 149-173. MR2336640 (2008f:18013) 
[14] Fei Xu, Hochschild and ordinary cohomology rings of small categories, Adv. Math. 219 (2008), no. 6, 1872-1893, DOI 10.1016/j.aim.2008.07.014. MR2455628(2009h:18025)

[15] F. Xu, Tensor structure on kC-mod and cohomology, Proc. Edinb. Math. Soc. (2) 56 (2013), no. 1, 349-370. MR3021416

[16] Fei Xu, On local categories of finite groups, Math. Z. 272 (2012), no. 3-4, 1023-1036, DOI 10.1007/s00209-011-0971-y. MR2995153

Department of Mathematics, Shantou University, Shantou, Guangdong 515063 , People's Republic of China

E-mail address: fxu@stu.edu.cn 